\title{
Small Bowel Obstruction with a Transition Point in a Patient on Peritoneal Dialysis
}

\author{
Babak S. Jazayeri-Moghadass ${ }^{a}$ Ryan Sutherland ${ }^{b}$ Lakir D. Patel ${ }^{b}$ \\ Valeriu Cebotaru ${ }^{\mathrm{a}}$ \\ aDivision of Nephrology, Departments of Medicine, University of Maryland School of \\ Medicine, Baltimore, MD, USA; ${ }^{b}$ Department of Diagnostic Radiology and Nuclear Medicine, \\ University of Maryland School of Medicine, Baltimore, MD, USA
}

\section{Keywords}

Dialysis · End-stage renal disease $\cdot$ Peritoneal dialysis

\begin{abstract}
Small bowel obstruction (SBO) is a rare complication of peritoneal dialysis (PD) that is usually seen in patients with encapsulating peritoneal sclerosis. We present a case of SBO that was caused by mechanical obstruction from omental adhesions around the PD catheter. This is the case of 71-year-old female with end-stage renal disease who was recently started on PD and presented with recurrent syncopal episodes and altered mental status. During hospitalization, the patient began experiencing incomplete drainage of the PD solution. Abdominal computerized tomography revealed SBO with a transition point near the PD catheter. The patient then underwent laparoscopy, which revealed omental adhesions around the PD catheter near the obstruction area, but no adhesion of the intestine was observed. The adhesions were dissected by laparoscopy, and the PD catheter was removed. This case highlights the challenges of PD access.
\end{abstract}




\section{Introduction}

Peritoneal dialysis (PD) is an excellent dialysis modality for end-stage renal disease patients; PD can be offered via home treatment. Some PD complications can hinder its effectiveness and ultimately impact patient survival. PD complications can be divided into 3 broad categories: infectious, mechanical, and metabolic. Infectious and mechanical complications are often catheter related. Catheter infection can lead to peritonitis, whereas catheter-related mechanical complications can result in ineffective dialysis and ultrafiltration failure. Some mechanical complications are not catheter related, such as hernias, genital edema, hydrothorax, and back pain [1]. Small bowel obstruction (SBO) is a rare complication associated with PD. SBO is usually associated with encapsulating peritoneal sclerosis (EPS), a rare complication seen in PD patients [2,3]. We report a case of a PD patient without EPS who developed acute SBO with a transition point near the PD catheter.

\section{Case Report}

A 71-year-old female, with a past medical history of end-stage renal disease on PD, stroke, heart failure with reduced ejection fraction, COPD, atrial fibrillation, hypertension, type 2 diabetes mellitus, obesity, hyperlipidemia, and hysterectomy was admitted to our facility for recurrent syncopal episodes and altered mental status. The work-up for syncope included a head computerized tomography (CT) scan that showed no acute intracranial abnormality and an echocardiogram that showed mild left ventricular hypertrophy, a left ventricular ejection fraction of 45-50\%, and mild global hypokinesis. One month prior to admission, a doublecuffed PD catheter (Covidien) was placed laparoscopically without omentectomy. There were no complications during PD catheter placement. During the 2 weeks prior to admission, the patient underwent PD training 5 times weekly. During PD training, the patient had 2 exchanges over $3 \mathrm{~h}$ (1,000 mL/exchange) with a 1.5\% dextrose, $2.5 \mathrm{mEq}$ calcium solution. Initial laboratory tests revealed the following: BUN $56 \mathrm{mg} / \mathrm{dL}$, creatinine $5.55 \mathrm{mg} / \mathrm{dL}, \mathrm{WBC} 7,900 / \mu \mathrm{L}$, and hemoglobin $9.1 \mathrm{~g} / \mathrm{dL}$. The rest of the electrolytes were unremarkable. The patient was started on PD treatment via continuous cycling PD with a 1.5\% dextrose, $2.5 \mathrm{mEq}$ calcium solution (5 exchanges over $10 \mathrm{~h}$ with 2,000 mL/exchange). On day 6 , the patient started to have incomplete drainage of the PD solution. On day 7, an abdominal CT scan without contrast was ordered. This scan showed mildly dilated small bowel loops in the right lower quadrant without features of SBO (Fig. 1a, b). Despite adding heparin to the PD solutions and changing the treatment from continuous cycling PD to continuous ambulatory PD $(2,000 \mathrm{~mL}, 1.5 \%$ dextrose, $2.5 \mathrm{mEq}$ calcium solution every $6 \mathrm{~h}$ ), the patient continued to have incomplete drainage. On day 8, PD fluid was sent for analysis, revealing 50 WBCs with 69\% PMNs. PD fluid culture was negative. The PD fluid remained clear throughout admission. On day 10 , the patient started having nausea without vomiting or abdominal pain. On day 11, PD was stopped. On day 12, a second abdominal CT scan showed dilated, stacked loops of proximal small bowel adjacent to the PD catheter, indicating SBO with a transition point near the PD catheter (Fig. 1c, d), which represent a continuation of the lesions identified by the first CT scan. The patient was transitioned to hemodialysis and treated via a nasogastric tube with intermittent suctioning. She then underwent a diagnostic laparoscopy that revealed omental adhesions around the PD catheter near the obstruction area, but no adhesion of the intestine. The adhesions were dissected by laparoscopy, and the PD catheter was removed. The patient's condition improved after the surgery, and she was discharged to a rehabilitation facility on hemodialysis.

\section{Karger'}



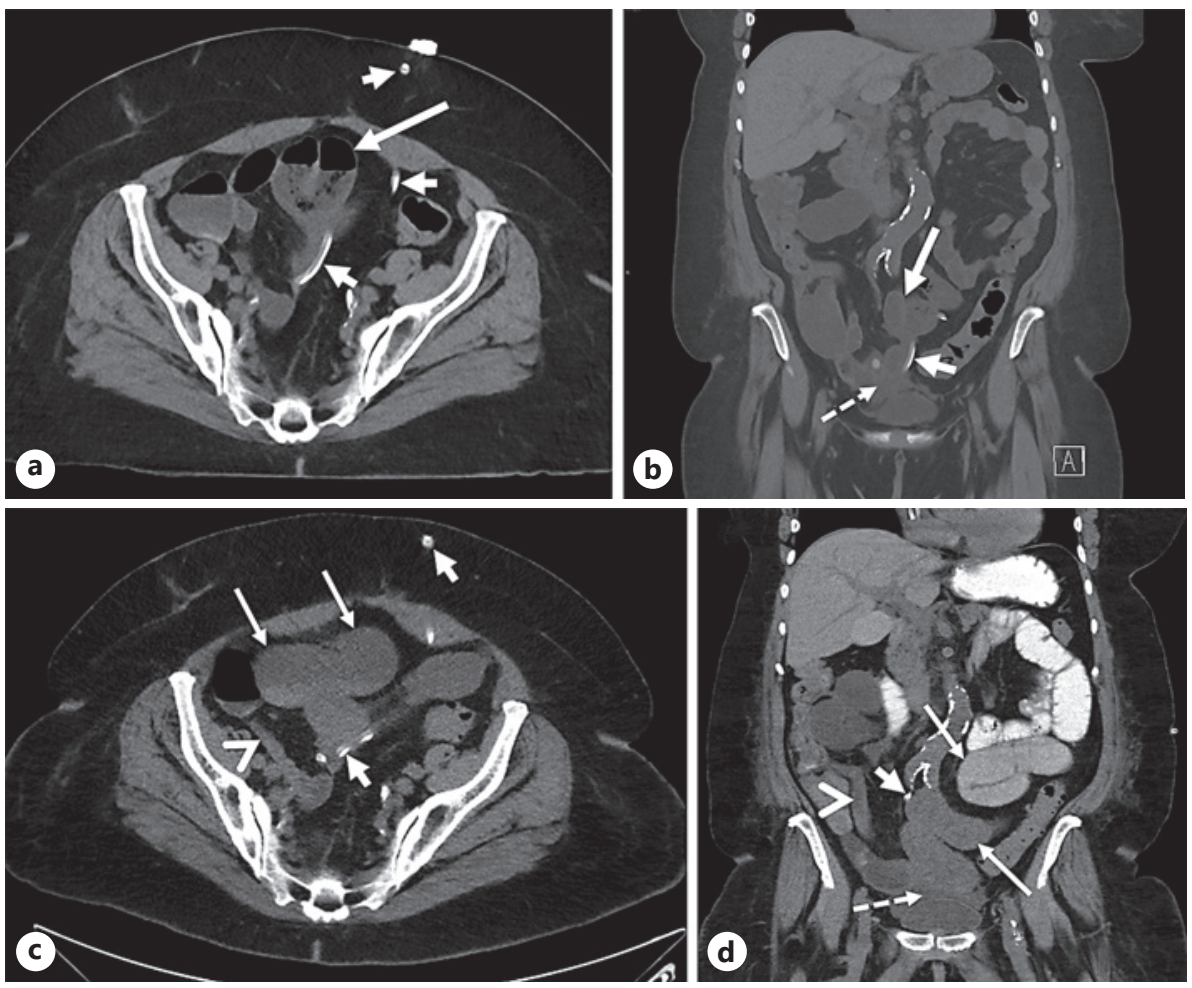

Fig. 1. SBO with a transition point. Axial (a) and coronal (b) images from CT abdomen and pelvis performed on day 7. Minimal adjacent free fluid is present in the pelvis (interrupted arrow, b). A prominent small bowel loop in the central abdomen (long arrow $\mathbf{a}, \mathbf{b}$ ) near the peritoneal catheter (short arrow) has intraluminal fluid and foci of gas, which could suggest small bowel fecalization, a sign of slowed intestinal transit. This loop is not dilated and there are no features of SBO. c Axial image taken on day 12 demonstrating dilated loops of proximal small bowel (long arrows) adjacent to the PD catheter (short arrows) with decompressed distal small bowel (arrowhead). d Coronal image taken on day 12 demonstrating dilated, stacked loops of proximal small bowel (long arrows) adjacent to the PD catheter (short arrow) with decompressed distal small bowel (arrowhead).

\section{Discussion}

SBO is a rare complication associated with $\mathrm{PD}$. The literature search revealed that $\mathrm{SBO}$ is commonly seen in EPS, a rare complication affecting PD patients [2]. Several cases of SBO have been described in PD patients without EPS. A case of bowel herniation through the peritoneal opening around the dialysis catheter has been associated with SBO [4]. Another case described in the literature involved SBO resulting from localized PD catheter-related adhesions in a patient who had been on PD for 3 years [5]. In that case, the omentum had formed fibrous adhesions around the catheter tip, leading to the formation of an internal hernia in which a small bowel loop had become caught.

We present a case of SBO with a transition point adjacent to the PD catheter that was caused by mechanical obstruction from omental adhesions around the PD catheter. The patient required laparoscopic intervention for adhesion dissection and catheter removal. Risk factors for SBO in PD patients include adhesions from prior abdominal surgery, EPS, abdominal hernias, and abdominal tumors. None of the described risk factors were present in this patient. It is also possible that the PD catheter or the biocompatibility of PD solutions caused local inflammation that could have led to omental adhesions. These outcomes are 
Case Reports

in Nephrology

and Dialysis

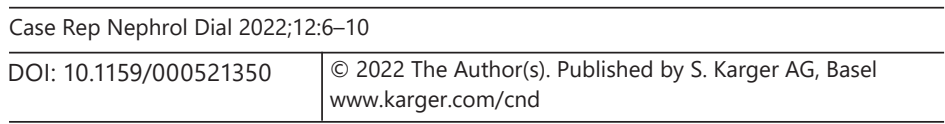

Jazayeri-Moghadass et al.: SBO Caused by PD Catheter

unlikely because no peritoneal inflammation was observed during laparoscopic removal of the PD catheter. To the best of our knowledge, this is a unique case that has not been previously described in the literature.

SBO can be added to the list of PD catheter-related complications, which includes leakage, infection, adhesion, migration, catheter obstruction, and hernia. One study showed that omentectomy at the time of PD catheter placement reduced the rate of complications from $27 \%$ to $7 \%$ in pediatric patients ranging in age from 1 day to 23 years [6]. The Canadian Association of Pediatric Nephrologists recommends discretionary omentectomy for PD catheter placement [7], whereas the International Society of Peritoneal Dialysis recommends omentopexy for redundant omentum when it is found within juxtaposition of the catheter tip [8]. This patient did not undergo omentectomy during laparoscopic PD placement, which might have prevented the formation of omental adhesions around the PD catheter and thus prevented SBO. This case highlights the challenges of PD access and suggests a potential benefit of omentectomy at the time of PD catheter placement.

\section{Statement of Ethics}

This study was conducted according to the guidelines laid down in the World Medical Association Declaration of Helsinki. Verbal informed consent was obtained via phone from the patient's daughter for publication of this case report and accompanying images. Patient had mild cognitive impairment and deferred all decisions to her daughter. This consent procedure (HP-00096137) was reviewed and approved by Human Research Protections Office (HRPO) at University of Maryland School of Medicine. University of Maryland School of Medicine HRPO does not require study protocols for case reports and therefore this paper is exempt from HRPO approval.

\section{Conflict of Interest Statement}

The authors have no conflicts of interest to declare.

\section{Funding Sources}

No funding was received for this study.

\section{Author Contributions}

Babak S. Jazayeri-Moghadass contributed to acquisition of data and wrote the first draft of the manuscript. Ryan Sutherland and Lakir D. Patel prepared figures. Valeriu Cebotaru provided supervision and mentorship and wrote the final draft of the manuscript. All the authors approved the final manuscript.

\section{Data Availability Statement}

All data generated or analyzed during this study are included in this article. Further inquiries can be directed to the corresponding author. 


\section{References}

1 McCormick BB, Bargman JM. Noninfectious complications of peritoneal dialysis: implications for patient and technique survival. J Am Soc Nephrol. 2007;18(12):3023-5.

2 Danford CJ, Lin SC, Smith MP, Wolf JL. Encapsulating peritoneal sclerosis. World J Gastroenterol. 2018;24(28): 3101-11.

3 Akbulut S. Accurate definition and management of idiopathic sclerosing encapsulating peritonitis. World J Gastroenterol. 2015;21(2):675-87.

4 Madden MA, Beirne GJ, Zimmerman SW, Sollinger H. Acute bowel obstruction: an unusual complication of chronic peritoneal dialysis. Am J Kidney Dis. 1982;1(4):219-21.

5 Hausmann MJ, Vorobiov M, Ovnat A. Small bowel obstruction due to localized Tenckhoff catheter-related adhesions. Perit Dial Int. 2002;22(3):431-3.

6 LaPlant MB, Saltzman DA, Segura BJ, Acton RD, Feltis BA, Hess DJ. Peritoneal dialysis catheter placement, outcomes and complications. Pediatr Surg Int. 2018;34(11):1239-44.

7 White CT, Gowrishankar M, Feber J, Yiu V, Canadian Association of Pediatric Nephrologists; Peritoneal Dialysis Working Group. Clinical practice guidelines for pediatric peritoneal dialysis. Pediatr Nephrol. 2006;21(8): 1059-66.

8 Crabtree JH, Shrestha BM, Chow KM, Figueiredo AE, Povlsen JV, Wilkie M, et al. Creating and maintaining optimal peritoneal dialysis access in the adult patient: 2019 update. Perit Dial Int. 2019;39(5):414-36. 